\title{
ALCOHOL CONSUMIDO Y VARIABLES ASOCIADAS EN BUCARAMANGA, COLOMBIA
}

\section{ALCOHOL CONSUMPTION AND ASSOCIATED VARIABLES IN BUCARAMANGA, COLOMBIA}

\author{
Oscar Fernando Herrán F. (1, 2), María Fernanda Ardila L. (2) \\ (1) Escuela de Nutrición y Dietética. Universidad Industrial de Santander, Colombia. \\ (2) Observatorio Epidemiológico de Enfermedades Cardiovasculares. Centro de Investigaciones Epidemiológicas. \\ Universidad Industrial de Santander, Bucaramanga, Colombia.
}

\begin{abstract}
The objective of this work was to estimate the quantity of consumed alcohol (g) by adults of Bucaramanga, and to describe the associated variables like problem drinking, alcoholism and characteristics of the consumer. During 2006 a total 1,119 subjects answered five questionnaires, including a food frequency questionnaire validated in this population. Men consumed $69.4 \mathrm{~g}$ weekly of alcohol (CI; 64.1 to 75.2) and women consumed $34.1 \mathrm{~g}$ (IC; 30.9 to 55.7), $p=0.000$. Fifty five \% of the population exceeded the recommended limit of weekly consumption (70.4\% of the men and 40.9\% of the women, $p=0.000$ ). The quantity of energy associated to the intake of alcohol was 781 kilocalories (CI; 696 at 894), of these 20\% (159 kilocalories, CI; 138 to 179) come from intake associated with soft drinks of the cola type. Six variables explain 33\% of the quantity of consumed alcohol; sex, age, socioeconomic level, consumption frequency, taste and problem drinking.

Key words: Alcohol drinking; epidemiology; diet; adverse effects; Colombia.
\end{abstract}

Este trabajo fue recibido el 17 de Marzo de 2009 y aceptado para ser publicado el 20 de Junio de 2009.

\section{INTRODUCCIÓN}

El consumo de alcohol ha sido asociado tanto positiva como negativamente con eventos de importancia para la salud pública (1-5); el riesgo de desarrollar cáncer en el tracto gastrointestinal, incluido el de hígado, aumenta con la cantidad de alcohol consumida $(6,7)$. En la enfermedad cardiovascular la ingesta acumulada de hasta 23,3 Unidades Internacionales de consumo semanal de alcohol (UI), tiene efecto protector, reduce hasta el $25 \%$ del riesgo de desarrollar enfermedad coronaria (8). Pero no sólo es importante la cantidad de alcohol consumida, también lo es el patrón de consumo (9). La ingesta regular y en dosis bajas como la reportada en la dieta denominada “mediterránea" es deseable (10-14), pero la ingesta elevada en ocasiones únicas hasta la embriaguez, está asociada entre otros eventos a violencia, accidentes con vehículo automotor, y otros que en poblaciones desarrolladas y en vías de desarrollo ya son epidémicos o endémicos $(9,15,16)$.

Muchos hallazgos controversiales con respecto al papel del alcohol en la salud pública, se deben a la mala medición de la cantidad consumida, y la poca especificidad de conceptos derivados, como el de consumidor problemático, el de alcohol dependiente, el de consumidor ceremonial, o la tipología del consumidor (9, 17-19). La medición correcta del consumo es compleja y exige de esfuerzos metodológicos que además, son costosos (20).

Los resultados aquí presentados son producto de la aplicación de instrumentos de medición desarrollados y validados en la Universidad Industrial de Santander (18-20, 21), con el objetivo de caracterizar adecuadamente la cantidad de alcohol consumida ( $\mathrm{g} /$ semana), los conceptos derivados como el de consumidor ceremonial, 
problemático y alcohólico, y algunas variables asociadas de tipo sociodemográfico, culturales y biológicas. Además, se discute sobre las implicaciones de los resultados sobre la salud pública. El estudio se realizó en una ciudad Colombiana donde el consumo se hace por ocasión hasta niveles tóxicos, y donde las tasas de mortalidad por neoplasias relacionadas con el consumo de alcohol, y de mortalidad por enfermedad isquémica del corazón son 1,54 y 1,42 veces mayores que las nacionales $(22,23)$.

\section{SUJETOS Y MÉTODO}

En el período 2006 - 2008, se realizó un estudio analítico tipo "cross-sectional" con base en una encuesta de prevalencia de corte transversal en una muestra representativa de la población adulta de Bucaramanga. Esta ciudad, es el principal polo de desarrollo del nororiente colombiano, su temperatura media es de $26^{\circ} \mathrm{C}$, y cuenta con 900000 habitantes.

\section{Tamaño de la muestra}

Mil ciento noventa y nueve sujetos fueron encuestados. Este tamaño fue definido como diez por el número de ítems del formato AEQ-III $(10 \times 120)(24,25)$, y permite con un $95 \%$ de confiabilidad representar en la población la cantidad de alcohol consumida, el consumo problemático y la dependencia del alcohol.

\section{Selección de sujetos}

Fueron seleccionados a través de muestreo en tres etapas para representar por sexo y estrato socioeconómico a la población. Los que cumplieron los siguientes criterios fueron elegibles; 1- Entre 18 y 60 años. 2Residir en la ciudad dos años ó más. 3- No asistir a un grupo de apoyo como alcohólicos anónimos y 4- Que manifestaran su deseo de participar suministrando voluntariamente la información.

De un mapa de Bucaramanga fueron elegidas al azar manzanas y luego casas por estrato socioeconómico, en cada vivienda se realizó un censo de elegibles y aleatoriamente se seleccionó uno por vivienda. Si esta persona se rehusaba, se elegía otra persona de la misma vivienda. Después de seleccionarlos, de informarlos sobre los objetivos, de garantizar la confidencialidad y de obtener un consentimiento escrito, se encuestaron. El proyecto y los procedimientos fueron aprobados por el comité de ética en investigación de salud de la Universidad Industrial de Santander.

\section{Instrumentos de medición}

Se aplicaron cinco en el siguiente orden; un cuestionario sobre aspectos biológicos, dietarios y sociode- mográficos, un cuestionario de frecuencia de consumo (CFC) para determinar la cantidad (g) de consumo semanal de alcohol durante el último año (20), la prueba de Cutting down, Annoyance by criticism, Gulty feeling, and Eye-openers (CAGE) para establecer la dependencia al alcohol (18), uno que permite establecer el consumo problemático diferente a la dependencia (CP-alcohol) (19) y finalmente la prueba AEQ-III, para establecer las expectativas positivas hacia el consumo de alcohol $(21,26)$.

\section{Variables}

Los estratos socioeconómicos 1 y 2, los más bajos en la escala de ingreso y desarrollo, se agruparon en el nivel socioeconómico 1 , los 3 y 4 en el nivel 2 y los estratos 5 y 6 en el nivel 3. El índice de masa corporal (IMC) se estableció como el peso $(\mathrm{kg})$ dividido por la talla elevada al cuadrado (mts2), y agrupado utilizando puntos de corte conocidos internacionalmente. Los sujetos que se declararon abstemios o consumidores ceremoniales, es decir que sólo consumen un trago o cerveza máximo, esporádicamente, y siempre en contextos de celebraciones, no fueron considerados consumidores de alcohol (19). Los gramos de alcohol estimados con el CFC, permitieron establecer unidades internacionales de consumo [g de alcohol/10], y generar una variable dicotómica con base en el límite máximo recomendado como seguro de consumo semanal de alcohol en UI, que para los hombres es $<=28$, y para las mujeres $<=17$ (27). El consumo problemático diferente a la dependencia fue declarado con una respuesta positiva o más en la prueba CP-alcohol (19) y la dependencia con tres ó más respuestas positivas en la prueba de CAGE (18). Con lo preestablecido para esta población se derivaron del AEQ-III cuatro expectativas hacia el consumo de alcohol (21); "incremento de la expresividad y la sexualidad", "disminución de la tensión física", "desinhibición y sentimientos de poder" y "disminución de la tensión psicológica".

Finalmente, se establecieron tres tipologías de consumidor; tipo A: Sujetos que no exceden el límite de consumo en unidades internacionales y son negativos para CP-alcohol y CAGE, tipo B: Que exceden el límite de consumo en unidades internacionales y son negativos para CP-alcohol y CAGE y tipo C: Son positivos para CP-alcohol y/o CAGE, sin importar si excede o no el límite de consumo en unidades internacionales.

\section{Análisis estadístico}

Dada la asimetría en el consumo semanal de alcohol (g), esta variable fue transformada a su logaritmo natural. El análisis se realizó en dos etapas, primero se describie- 
ron las variables con el fin de caracterizar la población y su consumo de alcohol, utilizando promedios, errores estándar y percentiles, y pruebas de $\mathrm{t}$ de studen, $\mathrm{Ji}^{2}$ y ANOVA y reportando intervalos de confianza del 95\% (IC). La segunda etapa permitió establecer qué variables están asociadas a la cantidad de alcohol consumido, para ello se utilizó un modelo lineal múltiple. Las variables fueron candidatas para entrar al modelo si en una relación bivariada con la cantidad de alcohol consumido, alcanzaban un valor de $\mathrm{p}<=0,20$ (28). Para el modelo final se estableció la bondad de su ajuste a través del cálculo del estadístico $\mathrm{r}^{2} \mathrm{o}$ coeficiente de determinación.

La información fue sometida a doble digitación y validada en EpiInfo v 6.04d (29). Dado el diseño de la muestra la estimación de los errores se realizó utilizando el ajuste de la varianza de Taylor $(30,31)$. El procesamiento de las variables y los cálculos estadísticos se realizaron con STATA/SE versión 10.1 (32).

\section{RESULTADOS}

\section{Características de la población estudiada}

Todos los sujetos contestaron las cinco encuestas, de ellos 611 (51\%) fueron mujeres. La edad media fue de 35,9 años (IC; 35,2 a 36,6), sin diferencia por sexo ( $\mathrm{p}=0,227)$. Entre 195 y 206 sujetos participaron por cada uno de los seis estratos socioeconómicos originales, $33 \%$ de la muestra en los niveles socioeconómicos uno y tres respectivamente. La escolaridad media fue de 11,5 años (IC; 11,3 a 11,8), el 1,5\% de la población no alcanzó la educación básica primaria. El IMC promedio en los hombres fue de 24,9 (IC; 24,4 a 25,4) y en las mujeres de 24,5 (IC; 24,1 a 24,9), p=0,147. El 46,1\% de los hombres y el $39,4 \%$ de las mujeres tienen IMC por encima de 25, $\mathrm{p}=0,001$. El $21 \%$ de los hombres y el $32 \%$ de las mujeres reconocieron haber practicado algún tipo de dieta en el último año, $\mathrm{p}=0,000$, sólo el 49,6\% de esta práctica fue recomendada por un médico o nutricionista. Según CP-alcohol, 123 hombres (21\%) y 44 mujeres $(7,2 \%)$ tienen consumo problemático diferente a la dependencia, $\mathrm{p}=0,000$. Según CAGE, 91 hombres $(15,5 \%)$ y $26 \mathrm{mu}-$ jeres $(4,3 \%)$ tienen riesgo alto de alcoholismo, $p=0,000$. El 13,9\% de la población tiene consumo problemático y el 9,8\% riesgo alto de alcoholismo. El 68\% de la población consumió regularmente alcohol durante el último año, $82,8 \%$ de los hombres y $53,7 \%$ de las mujeres, $\mathrm{p}=0,000$. El $26,7 \%$ de los hombres y el $57,1 \%$ de las mujeres presentan tipología de consumo A, el 45,9\% de los hombres y el 33,5\% de las mujeres tipología B y el $27,4 \%$ y $9,4 \%$ de hombres y mujeres respectivamente, tipología de consumo $\mathrm{C}, \mathrm{p}=0,000$. Un total de 739 sujetos consumió alcohol regularmente durante el último año, la edad se relacionó de manera inversa con la cantidad de alcohol consumido, así como la escolaridad, el nivel socioeconómico y el gusto por las bebidas alcohólicas, $p<0,001$. La frecuencia de consumo se relaciona de manera directa con la cantidad consumida, $\mathrm{p}<0,001$. La tabla 1 presenta las características de la población consumidora, y la cantidad de alcohol consumida por ésta (g/semana).

\section{Cantidad semanal de alcohol consumido}

Los hombres consumen en promedio $69,4 \mathrm{~g}$ de alcohol (IC; 64,1 a 75,2), las mujeres 34,1 g (IC; 30,9 a 55,7$), p=0,000$. El $55,4 \%$ de la población excede el límite de consumo semanal en unidades internacionales; $70,4 \%$ los hombres y $40,9 \%$ las mujeres respectivamente, $\mathrm{p}=0,000$. Las personas que reconocen practicar dieta consumen en promedio $9 \mathrm{~g}$ menos de alcohol (IC; 0,7 a $0,10)$ que aquellas que no, $\mathrm{p}=0,026$, el consumo en los sujetos clasificados con tipología C es de 83,9 g (IC; 73,0 a 95,6), en los consumidores tipo B de 51,9 g (IC; 48,4 a 55,7) y en los consumidores tipo A de 13,5 g (IC; 12,8 a 14,3), $\mathrm{p}=0,000$. Los que reconocen mucho gusto por el trago consumen $122,7 \mathrm{~g}$ de alcohol por semana (IC; 96,5 a 156,0), los que reconocen gusto moderado $62,2 \mathrm{~g}$ (IC; 56,3 a 68,0), y los que reconocen poco gusto $36,2 \mathrm{~g}$ (IC; 33, 1 a 40,0), p=0,000. Los sujetos que reconocen consumir alcohol con una frecuencia semanal en promedio ingieren 131,6 g (IC; 113,3 a 148,4), los que consumen cada quince días 69,4 g (IC; 59,7 a 79,8), y los sujetos que consumen mensualmente en promedio ingieren 37,3 g (IC; 34,8 a 40,4), p=0,000. La cantidad media de energía asociada a la ingesta de alcohol es de 781 kilocalorías (IC; 696 a 894), de estas el 20\%; 159 kilocalorías (IC; 138 a 179) son derivadas de bebidas acompañantes, como las tipo kola. Los hombres consumen 2,7 más kilocalorías derivadas de bebidas acompañantes que las mujeres. El 21,2\% (156) de los sujetos consumidores presentó consumo problemático,

\section{Consumo problemático y cantidad consumida}

Los sujetos que durante el último año reconocieron tener discusiones acaloradas con un familiar, amigo, o desconocido bajo el efecto del alcohol, consumen el doble de alcohol que aquellos que no, en los que el consumo es de; 47,5 g (IC; 44,2 a 50,4), p=0,000. Los sujetos que durante el último años se vieron involucrados en problemas bajo los efectos del alcohol que requirieron la presencia de la policía u otra autoridad, consumen 2,4 veces más cantidad de alcohol que aquellos que no, en los que el consumo es de 50,4 g (IC; 46,5 a 53,5), p=0,000. Los sujetos que en el último año se vieron involucrados en accidentes de tránsito bajo los efectos del alcohol consumen 2,6 veces más alcohol que aquellos que no, 
en los que es de 50,9 g (IC; 47,5 a 54,0), p=0,000. Los sujetos que durante el último año propiciaron discusiones familiares bajo los efectos del alcohol con arrepentimiento posterior, consumen el doble del alcohol que aquellos que no, en los que es de 49,9 g (IC; 46,5 a 53,5), p=0,000. Los sujetos que durante el último año y bajo los efectos del alcohol se vieron involucrados en alguna situación con componente erótico ó sexual por la cual se sintieron mal o se arrepintieron posteriormente, consumen 2,1 más alcohol que aquellos que no, en los que es de 48,9 g (IC;
45,6 a 52,5), p=0,000. En 156 de los 167 sujetos con consumo problemático se pudo establecer la cantidad de alcohol consumida en el último año ( $\mathrm{g} / \mathrm{semana})$, la cantidad consumida por éstos es 1,89 veces mayor que en aquellos sin consumo problemático; $85,4 \mathrm{~g} /$ semana versus 45.1 respectivamente (tabla 2 ).

\section{Riesgo de alcoholismo y cantidad consumida}

Los sujetos que en el último año sintieron la necesidad de disminuir la cantidad de alcohol que toman,

\section{TABLA 1}

\section{Características por sexo de la población consumidora de alcohol y cantidad consumida de alcohol semanalmente.}

\begin{tabular}{|c|c|c|c|c|}
\hline \multirow[b]{2}{*}{ Variable $\dagger$} & \multicolumn{4}{|c|}{ Alcohol consumido (g/semana) } \\
\hline & $\mathbf{n}$ & Hombre & $\mathbf{n}$ & Mujer \\
\hline \multicolumn{5}{|l|}{ Edad (años) } \\
\hline Hasta 24 & 143 & $93,7(80.6$ a 107,8$) *$ & 94 & $42,5(35,5$ a 50,9$)$ \\
\hline 25 a 39 & 158 & $66,7(58,6$ a 75,9$)$ & 107 & $33,8(28,8$ a 39,2$)$ \\
\hline 40 y más & 141 & $54,0(46,5$ a 22,9$)$ & 96 & $28,2(23,3$ a 33,8$)$ \\
\hline \multicolumn{5}{|l|}{ Escolaridad (años) } \\
\hline Hasta primaria & 24 & $60,3(40,4$ a 89,1$)$ & 13 & $18,9(16,1$ a 22,4$)$ \\
\hline Hasta secundaria & 74 & $53,5(45,6$ a 63,4$)$ & 66 & $31,2(25,3$ a 38,1$)$ \\
\hline Más de secundaria & 344 & $74,4(67,4$ a 81,4$)$ & 218 & $36,6(32,5$ a 40,8$)$ \\
\hline \multicolumn{5}{|l|}{ Nivel socioeconómico } \\
\hline Uno & 124 & $58,6(50,9$ a 67,4$)$ & 80 & $31,2(26,6$ a 36,6$)$ \\
\hline Dos & 153 & $74,4(64,1$ a 90,0$)$ & 102 & $30,9(26,3$ a 36,2$)$ \\
\hline Tres & 165 & $73,7(66,7$ a 81,4$)$ & 115 & $39,6(33,1$ a 47,5$)$ \\
\hline \multicolumn{5}{|l|}{ Gusto por el trago } \\
\hline Poco & 155 & $50,4(44,2$ a 57,4$)$ & 165 & $26,8(24,0$ a 30,3$)$ \\
\hline Moderado & 236 & $74,4(67,4$ a 83,1$)$ & 118 & $42,5(36,2$ a 49,4$)$ \\
\hline Mucho & 51 & $131,6(101,5$ a 169,0$)$ & 14 & $94,6(48,4$ a 184,9$)$ \\
\hline \multicolumn{5}{|l|}{ Frecuencia de consumo } \\
\hline Semanal & 112 & $139,8(119,1$ a 164,0$)$ & 25 & $97,5(66,0$ a 142,6$)$ \\
\hline Quincenal & 86 & $75,2(66,7$ a 90,0$)$ & 33 & $55,7(41,7$ a 74,4$)$ \\
\hline Mensual & 244 & $49,4(44,7$ a 54,6$)$ & 239 & $28,8(26,0$ a 31,5$)$ \\
\hline \multicolumn{5}{|l|}{ Consumo Problemático $\ddagger$} \\
\hline No & 325 & $60,3(54,6$ a 66,0$)$ & 258 & $31,8(28,5$ a 35,2$)$ \\
\hline $\mathrm{Si}$ & 117 & $103,5(86,5$ a 124,0$)$ & 39 & $56,8(41,7$ a 77,5$)$ \\
\hline \multicolumn{5}{|l|}{ Riesgo de alcoholismo I } \\
\hline No & 361 & $65,4(59,7$ a 71,5$)$ & 276 & $32,5(29,4$ a 35,9$)$ \\
\hline $\mathrm{Si}$ & 81 & $90,0(74,4$ a 109,4$)$ & 21 & $64,7(40,4$ a 103,5$)$ \\
\hline
\end{tabular}




\section{TABLA 2}

Consumo semanal de alcohol (g); ajustado por edad, índice de masa corporal y escolaridad en adultos de Bucaramanga, Colombia.

\begin{tabular}{|c|c|c|c|c|c|c|c|c|c|}
\hline Variable & $\mathbf{n}$ & Promedio (EE)* & p5 & p10 & p25 & p50 & p75 & p90 & p95 \\
\hline \multicolumn{10}{|l|}{$\operatorname{Sexo} \dagger$} \\
\hline Todos & 739 & $52,2(1,0)$ & 56 & 65 & 76 & 93 & 103 & 109 & 112 \\
\hline Hombre & 442 & $69,4(1,0)$ & 21 & 32 & 32 & 64 & 112 & 247 & 324 \\
\hline Mujer & 297 & $34,1(1,0)$ & 12 & 12 & 19 & 25 & 55 & 119 & 243 \\
\hline \multicolumn{10}{|c|}{ Nivel socioeconómico } \\
\hline Uno & 204 & $46,5(1,1)$ & 19 & 19 & 29 & 42 & 67 & 159 & 221 \\
\hline Dos & 255 & $52,5(1,1)$ & 12 & 19 & 32 & 42 & 87 & 226 & 354 \\
\hline Tres & 280 & $57,0(1,1)$ & 12 & 12 & 32 & 54 & 111 & 249 & 294 \\
\hline \multicolumn{10}{|c|}{ Gusto por el trago $\dagger$} \\
\hline Poco & 320 & $37,7(1,0)$ & 12 & 12 & 19 & 32 & 52 & 114 & 249 \\
\hline Moderado & 354 & $60,3(1,0)$ & 19 & 19 & 32 & 60 & 99 & 214 & 295 \\
\hline Mucho & 65 & $118,6(1,1)$ & 25 & 32 & 67 & 121 & 246 & 354 & 782 \\
\hline \multicolumn{10}{|c|}{ Frecuencia de consumo ${ }^{\dagger}$} \\
\hline Semanal & 136 & $130.7(1,1)$ & 32 & 42 & 77 & 121 & 247 & 431 & 528 \\
\hline Quincenal & 118 & $66,5(1,1)$ & 19 & 26 & 42 & 66 & 119 & 235 & 295 \\
\hline Mensual & 483 & $38.1(1,0)$ & 12 & 12 & 19 & 32 & 60 & 101 & 165 \\
\hline \multicolumn{10}{|c|}{ Consumo Problemático †+ } \\
\hline No & 581 & $45.1(1,0)$ & 12 & 17 & 25 & 39 & 77 & 159 & 253 \\
\hline $\mathrm{Si}$ & 156 & $85,4(1,1)$ & 19 & 25 & 42 & 85 & 182 & 323 & 499 \\
\hline \multicolumn{10}{|c|}{ Riesgo de alcoholismo } \\
\hline Bajo & 635 & $48,4(1,0)$ & 12 & 19 & 25 & 42 & 85 & 186 & 295 \\
\hline Alto & 102 & $85.1(1,1)$ & 21 & 32 & 42 & 77 & 178 & 260 & 301 \\
\hline
\end{tabular}

Expectativas hacia el consumo de alcohol $\$$

Incremento de la expresividad y la sexualidad

$\begin{array}{llllllllll}\text { No } & 466 & 51.9(1,0) & 12 & 19 & 32 & 43 & 87 & 214 & 302 \\ \mathrm{Si} & 271 & 53,0(1,1) & 12 & 19 & 25 & 42 & 87 & 213 & 290\end{array}$

Disminución de la tensión física

$\begin{array}{lccccccccc}\text { No } & 362 & 50,2(1,0) & 12 & 19 & 32 & 42 & 88 & 211 & 297 \\ \mathrm{Si} & 375 & 54,4(1,0) & 12 & 19 & 32 & 42 & 85 & 221 & 291 \\ \text { esinhibición } & \text { y sentimientos de poder† } & & & & & & & \\ \text { No } & 107 & 43,6(1,1) & 12 & 12 & 19 & 42 & 67 & 138 & 328 \\ \mathrm{Si} & 330 & 54,0(1,0) & 12 & 19 & 32 & 42 & 89 & 223 & 295\end{array}$

Disminución de la tensión psicológica†

$\begin{array}{llllllllll}\text { No } & 310 & 47,7(1,0) & 12 & 12 & 25 & 42 & 85 & 159 & 288 \\ \mathrm{Si} & 427 & 55,7(1,0) & 12 & 19 & 32 & 43 & 89 & 235 & 301\end{array}$

* Con base en valores log.

$\dagger \mathrm{p}<0,05$.

$\ddagger$ Con base en la teoría de las expectativas $(21,26)$.

+ Con base en CP-Alcohol (19).

I Con base en CAGE (18)|

p; percentil. 
consumieron 1,5 veces más alcohol que aquellos que no, en los que el consumo es de 46, $1 \mathrm{~g}(42,5$ a 49,9), $\mathrm{p}=0,000$. Los sujetos que en el último año se han sentido molestos por que les critican su manera de beber, consumieron 1,6 más veces alcohol que aquellos que no, en los que es de 47,5 g (IC; 43,8 a 50,9), p=0,000. Los sujetos que en el último año se han sentido mal o culpables por su manera de beber, consumen 1,5 más alcohol que aquellos que no, en los que es de 47,0 g (IC; 43,8 a 50,9), p=0,000. Los sujetos que en el último año tomaron un trago en la mañana para calmar los nervios, consumen el doble de alcohol que aquellos que no, en los que es de 47,5 g (IC; 44,3 a 50,9), p=0,000.

\section{Expectativas positivas y cantidad consumida}

El consumo de alcohol fue diferencial para las siguientes expectativas; "desinhibición y sentimientos de poder" y "disminución de la tensión psicológica". Los sujetos positivos a las expectativas "incremento de la expresividad y la sexualidad" y "disminución de la tensión física", no presentaron consumo diferencial con los clasificados como negativos a éstas. Sin embargo, en las expectativas donde se evidenció esta diferencia sólo es de aproximadamente $10 \mathrm{~g} / \mathrm{semana}$ (tabla 2). Las tablas 1 a 3 presentan otros detalles de la cantidad consumida semanalmente de alcohol (g).

\section{Bebidas preferidas y cantidad consumida}

La cerveza es la bebida preferida, el $50,7 \%$ de la muestra la consume, le siguen el whisky y el vino con $17,8 \%$ y $17,1 \%$ respectivamente, el aguardiente lo con- sume el 14,3\% de la muestra y el ron el 10,3\%. Entre los consumidores, la cantidad media de alcohol por semana y derivada de la cerveza es de 57,8 $\mathrm{g}$ (IC; 50,6 a 65,0), la derivada del whisky $39,2 \mathrm{~g}$ (IC; 27,9 a 50,4), la derivada del vino de 37,4 $\mathrm{g}$ (IC; 25,9 a 43,6), la del aguardiente de $53,4 \mathrm{~g}$ (IC; 43,1 a 63,7), y la derivada del ron de 47,4 g $(41,3$ a 53,5). La tabla 3 presenta la cantidad de alcohol consumida por sexo y tipología del consumidor.

\section{Variables asociadas al consumo de alcohol}

Seis variables explican en un modelo lineal múltiple la cantidad de alcohol consumida; la frecuencia de consumo y la edad en relación inversa, el nivel socioeconómico, el gusto manifestado por el trago, ser hombre y el consumo problemático en relación directa. El coeficiente de determinación alcanzado por el modelo fue de 0,33 . La tabla 4 muestra los coeficientes parciales de regresión con sus IC del 95\%.

\section{DISCUSIÓN}

La proporción de hombres y mujeres estudiados, como la de sujetos sin educación básica primaria, se correspondió con la de la población general (33). Además, la prevalencia de riesgo de alcoholismo según CAGE y de consumo problemático fue similar a la reportada en estudios previos en la misma población (18, 19). Todo lo anterior permite suponer que la muestra representó a la población de adultos satisfactoriamente. La principal dificultad al relacionar la cantidad de alcohol consumida con eventos cardiovasculares y otros crónicos es la forma como esta es estimada, los

\section{TABLA 3}

\section{Consumo semanal de alcohol (g) por tipo de bebida, según tipología de consumo y sexo, en adultos de Bucaramanga, Colombia.}

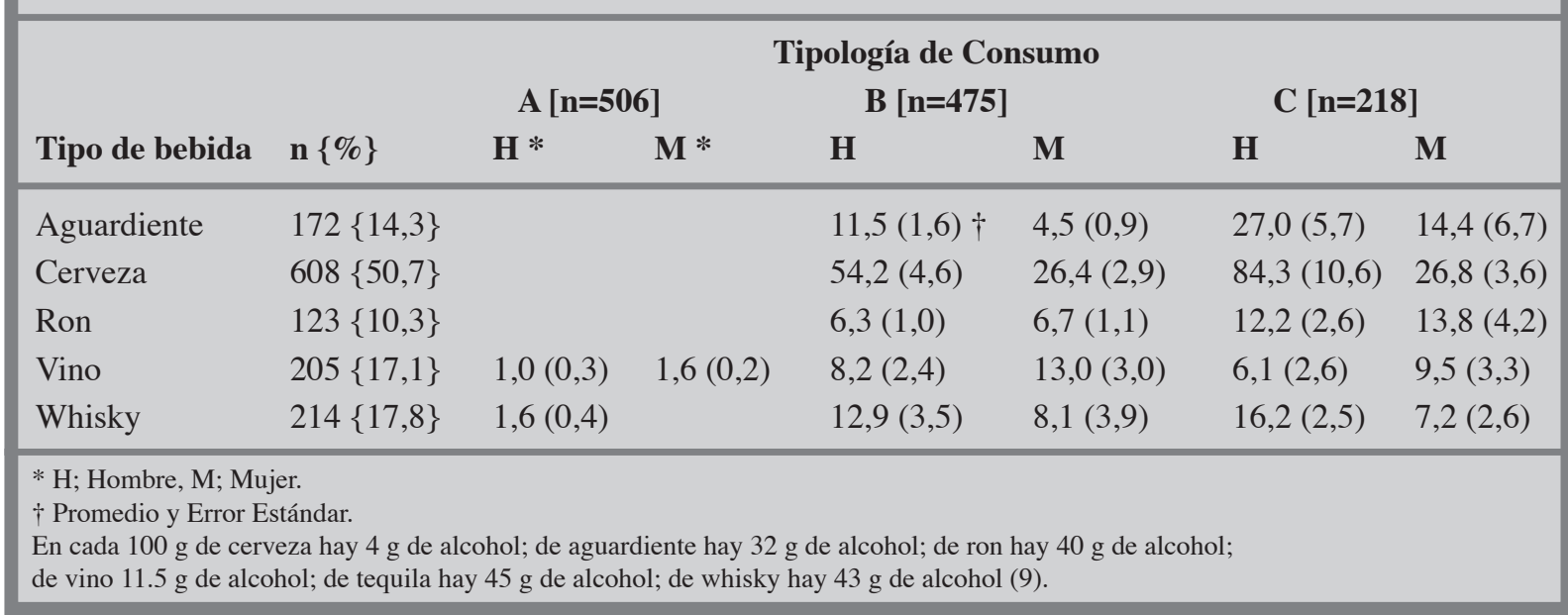


formatos de frecuencia de consumo han demostrado ser hasta ahora la mejor manera de cuantificar el consumo de alcohol $(2,20,34)$, esa es la principal fortaleza de este estudio.

El consumo de alcohol como previamente se había reportado (9), sigue haciéndose en niveles tóxicos, en ocasiones únicas y no está ligado a la dieta o consumo de alimentos, lo que no representa ningún efecto protector en el desarrollo de las enfermedades crónicas, en particular, de la cerebrovasculares y cardiovasculares, y por el contrario, genera problemas de salud pública (35), como los descritos en cada uno de los ítems analizados de las pruebas CP-alcohol y CAGE $(18,19)$.

Al hacer más precisa la definición de los sujetos consumidores, es decir, cuando se clasifican los consumidores ceremoniales como abstemios (19), la proporción de consumidores habituales disminuye, y en teoría la cantidad consumida aumenta, este hecho si no es considerado, diluye en modelos de regresión la asociación con los eventos de interés para la salud pública (36). Ya que la proporción de abstemios y consumidores ceremoniales es alta, es deseable considerar este aspecto metodológico como obligatorio en futuros estudios.

Ni la escolaridad, ni el IMC, se asociaron a la cantidad consumida de alcohol a pesar de la importante cantidad de kilocalorías derivadas, hallazgo ya reportado en esta población y que contradice otros informes $(9,37)$. Sin embargo, el autoreporte de la frecuencia de consumo y el gusto por el trago, junto con el nivel socioeconómico, el sexo, la edad y el consumo problemático sí, en un modelo anterior para explicar el consumo en esta población se había alcanzado un $\mathrm{r}^{2}$ de 17\% (9), el presentado alcanzó un $\mathrm{r}^{2}$ de $33 \%$, que si bien sigue siendo bajo, es el doble del anterior, lo que se explicaría

\section{TABLA 4}

\section{Coeficientes de regresión, con base en un modelo de regresión lineal múltiple para explicar la cantidad de alcohol consumido (g).}

$\mathrm{n}=1193$

Variable dependiente; log (Cantidad de alcohol consumida en gramos)

Coeficiente $\mathrm{r}^{2}$ ajustado; 0,33

\section{Variable}

Edad (Años)

Sexo

Mujer

Hombre

Nivel socioeconómico

Uno

Dos

Tres

\section{Coeficiente}

$-0,04(-0,06$ a $-0,02) *$

$+$

$1,62(1,10$ a 2,14$)$

Frecuencia de consumo

Semanal

Quincenal

$$
+
$$

$$
1,95(1,33 \text { a } 2,47)
$$

Mensual

Gusto por el trago

Poco

Moderado

Mucho

Consumo problemático $\ddagger$

No 
por los desarrollos metodológicos y en la medición aquí incorporados (18-21). En esta población se había demostrado que las expectativas positivas hacia el consumo son predictoras de la decisión de consumir alcohol, del consumo problemático y del riesgo de alcoholismo (38), a pesar de encontrar asociación en la relación bivariada para dos de ellas con la cantidad consumida, en el modelo multivariado ésta no se conservó, hallazgo hasta ahora no reportado.

La cantidad de alcohol consumida estuvo ligada lógicamente con las variables estudiadas; aumenta conforme la tipología de consumo representa mayor riesgo, es mayor conforme aumenta el riesgo de consumo problemático y el de alcoholismo, es mayor a menor intervalo entre consumos y mayor gusto auto reportado por el trago. Lo anterior se constituye en otro aspecto de la validez externa del CFC con el que se estimó el consumo (20).

La cerveza y el aguardiente siguen siendo las bebidas preferidas, pero llama la atención cómo la proporción de consumidores de vino y de whisky se ha incrementado en más del 300\% con respecto a la anterior medición (9). La fuente del alcohol puede tener implicaciones sobre la cantidad de energía consumida, la cantidad de nutrientes y otros factores nutricionales a los que se les confiere efectos protectores $(9,35)$. Por lo aquí presentado, valdría indagar a profundidad si también se relaciona con la tipología del consumidor, pues el vino es asociado a la dieta mediterránea y junto con el whisky en esta población, son consideradas como bebidas "saludables" y a veces "permitidas", incluso para sujetos diabéticos e hipertensos. Dos posibles explicaciones para el aumento de este tipo de bebidas a expensas de la cerveza y el aguardiente son, de un lado que los supermercados han promocionado agresivamente en los últimos cinco años el consumo de vino, y de otro que los impuestos para las bebidas como el whisky han disminuido como una medida para desestimular el contrabando, los datos recolectados no permiten confirmar lo anterior.

La prevalencia de riesgo de alcoholismo reportada, es 1,4 veces mayor que la del país; $7,1 \%(40,41)$, y la de consumo problemático diferente a la dependencia el doble $(39,40)$, la relación de consumidores [hombre/ mujer] es menor, 3,5 versus $6,0(9,39,40)$, lo que sumado con los niveles tóxicos de consumo, hacen a esta población vulnerable y candidata para implementar programas de intervención, que tengan como objetivos centrales el retardo en la edad de inicio en el consumo y una vez iniciado, la racionalización del mismo. Con la información acumulada hasta ahora en esta población, las intervenciones pueden ser muy específicas, tanto en los determinantes, como en la estimación de las líneas de base, incluida la cantidad de alcohol consumida, lo que permitiría evaluar el impacto de las mismas.

\section{RESUMEN}

El objetivo fue estimar la cantidad de alcohol consumida (g) por adultos de Bucaramanga, y describir el consumo por las variables derivadas del mismo; consumo problemático, riesgo de alcoholismo y tipología del consumidor. Durante 2006 un total 1.119 sujetos respondieron cinco encuestas, entre ellas, un cuestionario de frecuencia de consumo validado en esta población. Los hombres consumen en promedio 69,4 g semanales de alcohol (IC; 64,1 a 75,2), las mujeres 34,1 g (IC; 30,9 a 55,7), p=0,000. El 55,4\% de la población excede el límite de consumo semanal en unidades internacionales; $70,4 \%$ los hombres y $40,9 \%$ las mujeres respectivamente, $\mathrm{p}=0,000$. La cantidad de energía asociada a la ingesta de alcohol fue de 781 kilocalorías (IC; 696 a 894), de estas el 20\% (159 kilocalorías, IC; 138 a 179) son derivadas de bebidas acompañantes, como las tipo cola. Seis variables explican en $33 \%$ la cantidad de alcohol consumido; el sexo, la edad, el nivel socioeconómico, la frecuencia de consumo, el gusto por las bebidas y el consumo problemático.

Palabras clave: Consumo de bebidas alcohólicas; dieta; epidemiología; efectos adversos; Colombia.

\section{Dirigir la correspondencia a:}

Profesor

Oscar Fernando Herrán Falla. ND. MSc

Carrera 32 No. 29-31

Facultad de Salud. CIE.

Tercer Piso. Oficina 304

Universidad Industrial de Santander

Bucaramanga

Colombia.

Sur América

Tele fax; (57-7) 6345781 / 6323215

e-mail: herran@uis.edu.co

oscar.herran@gmail.com

Declaración de conflicto de interés: Los autores declaran que no tienen conflicto de interés de ningún tipo, ni real o potencial sobre los resultados presentados.

Financiamiento: El presente trabajo fue cofinanciado por la Universidad Industrial de Santander y el Instituto Colombiano para el Desarrollo de la Ciencia y la Tecnología "Francisco José de Caldas", COLCIENCIAS. El proyecto fue seleccionado mediante convocatoria pública y asignado el código 1102-345-18973. Código interno UIS; 8656. 


\section{BIBLIOGRAFÍA}

1. Haver B, Gjestad R, Lindberg S, Franck J. Mortality risk up to 25 years after initiation of treatment among 420 Swedish women with alcohol addiction. Addiction 2009;104:413-9.

2. Murray RP, Connett JE, Tyas SL, Bond R, Ekuma O, Silversides CK, Barnes GE. Alcohol Volume, Drinking Pattern, and Cardiovascular Disease Morbidity and Mortality: Is There a U-shaped Function?. Am J Epidemiol 2002;155:242-8.

3. Rehm J, Greenfield TK, Rogers JD. Average volume of alcohol consumption, patterns of drinking, and all-cause mortality: results from the US National Alcohol Survey. Am J Epidemiol 2001;153:64-71.

4. Corrao G, Rubbiati L, Bagnardi V, et al. Alcohol and coronary heart disease: a meta-analysis. Addiction 2000;95:1505-23.

5. Single E, Leino VE. The levels, pattern, and consequences of drinking. En: Grant M, Litvak J, eds. Drinking patterns and their consequences. Washington, DC: Taylor and Francis, 1998:7-24.

6. Gronbaek M, Becker U, Johansen D, Tonnesen H, Jensen G, Sorensen TI. Population based cohort study of the association between alcohol intake and cancer of the upper digestive tract. BMJ 1998; 317:844-48

7. Morgan TR, Mandayam S, Jamal MM. Alcohol and hepatocellular carcinoma. Gastroenterology 2004;127(5):S87-96

8. Rimm EB, Williams P, Fosher K, Criqui M, Stampfer MJ. Moderate alcohol intake and lower risk of coronary artery disease: Meta-analysis of effects on lipids and haemostatic factors. BMJ 1999; 319: 1523-28.

9. Herrán OF, Ardila MF. Consumo de alcohol, riesgo de alcoholismo y alcoholismo en Bucaramanga, Colomb Med 2005;36:158-67

10. Renaud S, de Lorgeril M. Wine, alcohol, platelets, and the French paradox for coronary heart disease. Lancet 1992;339:1523-6.

11. Criqui MH, Ringel BL. ¿Does diet or alcohol explain the French paradox? Lancet 1994;344:1719-23.

12. Muñoz MA, Fíto M, Marrugat J, Covas MI, Schröder $\mathrm{H}$. Adherence to the Mediterranean diet is associated with better mental and physical health. Br J Nutr 2008;15:1-7.

13. Sofi F, Cesari F, Abbate R, Gensini GF, Casini A. Adherence to Mediterranean diet and health status: meta-analysis. BMJ 2008;337;a1344.

14. Knoops KT, de Groot LC, Kromhout D, Perrin AE, Moreiras-Varela O, Menotti A, et al. Mediterranean diet, lifestyle factors, and 10-year mortality in el- derly European men and women: the HALE project. JAMA 2004;292:1433-9

15. López JL. Patrón de consumo de alcohol en pacientes captados en salas de urgencias. Salud Publica Mex 1998:40;487-93.

16. Luneta P, Penttila A, Sarna S. Water traffic accidents, drowning and alcohol in Finland, 1969-1995. Inter J Epidemiol 1998;27:1038-43.

17. Caetano R, Babor TF. Diagnosis of alcohol dependence in epidemiological surveys: an epidemic of youthful alcohol dependence or a case of measurement error? Addiction 2006;101(Suppl.1):111-4.

18. Herrán OF, Ardila MF. Validez interna y reproducibilidad de la prueba CAGE en Bucaramanga, Colombia. Biomédica 2005;25:231-41.

19. Herrán OF, Ardila MF. Consumo problemático de alcohol en Bucaramanga, Colombia: diseño y validación de una prueba. Biomédica 2008; 28:25-37

20. Herrán OF, Ardila MF. Validity and reproducibility of two semi-quantitative alcohol frequency questionnaires for Colombian population. Public Health Nutr 2006;9:763-70.

21. Herrán OF, Ardila MF. Validez factorial del cuestionario de expectativas hacia el consumo de alcohol (AEQ-III), en adultos de Bucaramanga, Colombia. Rev Méd Chile 2007;135: 488-95

22. Bautista L. Tendencias de la mortalidad por enfermedades cardiovasculares en Colombia y Santander, 1980-1996. Septiembre (Reporte Técnico). Bucaramanga: ICIB, UIS, PAHO; 2000.

23. Franco MC. La importancia de la promoción y prevención en la enfermedad cardiovascular. Rev Colomb Cardiol 2003:10;187-88.

24. Norman G, Streiner D. Bioestadística. Barcelona: Editorial Mosby, 1996;129-48.

25. Tavella NM. Análisis de los ítemes en la construcción de instrumentos psicométricos. México: Editorial Trillas, 1978;75.

26. Goldman MS, Del Boca FK, Darles J. Alcohol expectancy theory: The application of cognitive neuroscience. En: Leonard KE, Blane HT (Editors). Psychological theories of drinking and alcoholism. Second Edition. New York: Guilford Press, 1999:203-46.

27. González, J. Instituto Nacional de la Seguridad Social. Bilbao. Alcohol. Tipos de intervención en el consumo de riesgo. Disponible en: http://www. seslap.com/seslap/html/pubBiblio/revista/n_8/ n8Art2.pdf, Consultado el 20 de agosto de 2008.

28. Greenland S. Modeling and variable selection in epidemiologic analysis. Am J Public Health 1989; 79:340-49. 
29. CDC. Epilnfo, versión 6.04d. Epidemiología en ordenadores. Atlanta, Georgia. Enero, 2001.

30. STATA. Estimation of means, totals, ratios, and proportions for survey data. Stata Technical Bulletins 1996;6:213-35.

31. Levy SP, Lemeshow S. Sampling of populations: Methods and applications. 3th ed. New York: Jhon Wiley \& Sons; 1999:365-90.

32. StataCorp, 2008. Stata Statistical Software: Release 10.1 Collegue Station, TX: StataCorp LP.

33. PROFAMILIA. Encuesta Nacional de Demografía y Salud, 2005. Bogotá: Profamilia; 2005.

34. Willet W. Nutritional epidemiology. 2a ed. New York: Oxford University Press; 1998. p. 74-100.

35. Suter PM. Alcohol: su participación en la salud y la nutrición. En: Bowman AB, Russell RM (eds.). Conocimientos actuales, sobre nutrición. $8^{\mathrm{a}}$ ed. Publicación científica y técnica $\mathrm{N}^{\circ}$ 592. Washington,
DC: Organización Panamericana de la Salud: 2003. p. $543-554$.

36. Hamilton CL. Regression with graphics. A second couse in applied statistics. Belmont, California: Duxbury Press; 1992.

37. Wannamethee SG, Shaper AG. Alcohol, body weight, and weight gain in middle-aged men. Am J Clin Nutr 2003;77:1312-17.

38. Herrán OF, Ardila MF. Expectativas en el consumo de alcohol en Bucaramanga, Colombia. Rev Méd Chile 2007;135:1446-55.

39. Torres GY. Alcohol: Prevalencia de consumo y dependencia en Colombia. Rev Med CES 1999; 12:1-9.

40. Presidencia de la República. Ministerio de Salud. Programa Rumbos. Estudio Nacional sobre Uso y Abuso de Alcohol y Consumo de Sustancias Psicoactivas. Bogotá: Ministerio de Salud, 2000. 